\title{
Effects of Perioperative Rosuvastatin for Postoperative Delirium After Elderly General Anesthesia Patients: A Randomized, Double-Blind, and Placebo-Controlled Trial
}

\section{Xiaoqin Xu}

Wuxi Clinical College of Anhui Medical University, 904th Hospital of Joint Logistic Support Force of PLA Jingzhi Luo

Wuxi Clinical College of Anhui Medical University, 904th Hospital of Joint Logistic Support Force of PLA

Xiaoyu Li ( $\Delta 763256895 @ q q . c o m$ )

Wuxi Clinical College of Anhui Medical University, 904th Hospital of Joint Logistic Support Force of PLA Haiqin Tang

Wuxi Clinical College of Anhui Medical University, 904th Hospital of Joint Logistic Support Force of PLA Weihong Lu

Wuxi Clinical College of Anhui Medical University, 904th Hospital of Joint Logistic Support Force of PLA

\section{Research Article}

Keywords: perioperative rosuvastatin, postoperative delirium, elderly, general anesthesia, RCT

Posted Date: March 9th, 2021

DOI: https://doi.org/10.21203/rs.3.rs-182547/v1

License: (c) (i) This work is licensed under a Creative Commons Attribution 4.0 International License.

Read Full License 
Effects of perioperative rosuvastatin for postoperative delirium after elderly general anesthesia patients: a randomized, double-blind, and placebo-controlled trial

Running title: perioperative rosuvastatin for postoperative delirium

Xiaoqin Xu,MM; Jingzhi Luo, MM; Xiaoyu Li*, MM; Haiqin Tang, MM; Weihong Lu, MM

Department of Anesthesiology, Wuxi Clinical College of Anhui Medical University, $904^{\text {th }}$ Hospital of Joint Logistic Support Force of PLA, Wuxi Clinical College of Anhui Medical University, Wuxi 214000.

Address correspondence: Xiaoyu Li, Department of Anesthesiology, Wuxi Clinical College of Anhui Medical University, 904th Hospital of Joint Logistic Support Force of PLA, Xing Yuan North Road 101, Wuxi 214044, China. Tel: +86 13771166231; E-mail: 742888988@qq.com 


\begin{abstract}
Background: Experimental evidence has indicated the benefits of statins for the treatment of postoperative delirium. Previously clinical trials had no definite conclusions of statins on delirium. Some clinical trials indicated statins can reduce postoperative delirium and improve outcome, while some studies showed negative results. Therefore, the present study tries to evaluate whether perioperative rosuvastatin treatment could reduce the incidence of delirium and improve clinical outcomes.
\end{abstract}

Methods: This was a randomized, double-blind, and placebo-controlled trial in a single-center in Jiangsu, China. This study enrolled patients aged more than 60 years old who present selective general anesthesia operation, with informed consent. A computer-generated randomization sequence (in a 1:1 ratio) was used to randomly assign patients to receive either rosuvastatin (40 $\mathrm{mg} /$ day) or placebos. Participants, care providers, and investigators were all masked to group assignment. The primary endpoint was the incidence of delirium, assessed twice daily with the Confusion Assessment Method during the first 7 postoperative days. Analyses were performed by intention-to-treat and safety populations.

Results: Between Jan 1, 2017, and Jan 1, 2020, 3512 patients were assessed. A total of 821 were randomly assigned to receive either a placebo $(n=411)$ or rosuvastatin $(n=410)$. The incidence of postoperative delirium was significantly lower in the rosuvastatin group (23 [5.6\%] of 410 patients) than the placebo group (42 [13.5\%] of 411 patients $(\mathrm{OR}=0.522,95 \% \mathrm{CI}$ $0.308-0.885 ; \mathrm{p}<0.05)$. No significant difference on 30 -day all-cause mortality $(6.1 \%$ vs $8.7 \%$, OR 0.67, $\mathrm{P}=0.147,95 \%$ CI $0.39-1.2$ ) between two groups. Rosuvastatin can decrease the hospitalization time $(13.8 \pm 2.5$ vs $14.2 \pm 2.8, \mathrm{P}=0.03)$ and hospitalization expenses $(9.3 \pm 2.5$ vs $9.8 \pm 2.9, \mathrm{P}=0.007)$. No statistical significance between the two groups on abnormal liver enzymes (9.0\% vs $7.1 \%, \mathrm{P}=0.30, \mathrm{OR}=1.307,95 \%$ CI $0.787-2.169)$ and rhabdomyolysis $(0.73 \%$ vs $0.24 \%$, $\mathrm{P}=0.37, \mathrm{OR}=3.020,95 \%$ CI $0.31-29.2$.

Conclusion: The current study suggests that perioperative rosuvastatin treatment can reduce the incidence of delirium after elective general anesthesia operation. However, no evidence was found that rosuvastatin can improve clinical outcomes. The therapy was safe. Further investigation is necessary to fully understand the potential usefulness of dexmedetomidine in older patients.

Trial registration: chictr.org.cn, ChiCTR-IPR-17011984(Registered date: 13/07/2017). The 
manuscript adheres to CONSORT guidelines.

Key words: perioperative rosuvastatin; postoperative delirium; elderly; general anesthesia; RCT

\section{Introduction}

Delirium is a severe clinical syndrome characterized by a temporary organic mental disorder, acute brain dysfunction, changes in cognition, and disturbances in orientation, which may induce long-term cognitive impairment, death/disability, and increased length of hospital stay and costs[1]. Inouye[2] reported that postoperative delirium occurs in $11-51 \%$ of patients after surgery, and the rate is further increased in older patients. In the United States, more than 2.6 million adults older patients occurred delirium each year, accounting for an estimated annual health care expenditure of more than $\$ 164$ billion. Additionally, delirium or postoperative delirium may increase the long-term risk of dementia and mortality[3]. In the past 20 years, the treatment of delirium in older patients has become a severe challenge, haloperidol and ziprasidone were the most important drugs to treat delirium, but recent studies indicated that haloperidol and ziprasidone can not prevent delirium and the duration of delirium[4, 5]. Avidan[6] reported that Ketamine, a widely used anti-delirium drug also could not decrease delirium in older adults after major surgery, and on the contrary, it might cause harm by inducing negative experiences. Until now, there were no specific treatments and drugs for delirium.

Statins, as inhibitors of 3-hydroxy-3-methylglutaryl coenzyme A (HMG-CoA) reductase, are widely used in cardiovascular disorders as cholesterol-lowering medications. Recent studies had indicated that statins have been proven to exert pleiotropic effects, including anti-inflammatory, antioxidative stress, inhibit platelet aggregation, and neuroprotection[7-10]. As the neuroprotection related molecular mechanism was similar to the delirium, so it indicated that statins may prevent delirium. A retrospective, single-center study that included 1,132 vascular surgical patients confirmed that preoperative statins can significantly decrease the incidence of postoperative delirium[11]. While another observational cohort study included 4,659 consecutive patients after coronary revascularization at 2 university institutions concluded that preoperative statins did not decrease the incidence of delirium[12]. However, a very important and authoritative randomized controlled trial (NCT00979121 and NCT00719446) enrolled 568 patients (293/275) at 35 hospitals in the USA showed no benefit of rosuvastatin in reducing delirium or cognitive 
impairment in the Intensive Care Unit (ICU) during 12 months of follow-up[13]. There is a lack of proof from randomized controlled studies after elderly general anesthesia operative patients. Therefore, it is not clear whether rosuvastatin has preventive effects against delirium. The present study, therefore, explored whether acute rosuvastatin treatment could reduce postoperative delirium and improve clinical outcomes after general anesthesia operative patients

\section{Methods}

\section{Study design}

This was a randomized, double-blind, and parallel arm placebo-controlled trial of single-center institutions in Jiangsu, China, between Jan 1, 2017, and Jan 1, 2020, 3512 patients were assessed. Anhui Medical University Affiliated Wuxi Clinical college ( $904^{\text {th }}$ Hospital of PLA), was included. The study was registered in the chictr.org.cn with number: ChiCTR-IPR-17011984(Registered date: 13/07/2017). The study was designed to assess the superiority of intervention. The study protocol was approved by the Anhui Medical University Affiliated Wuxi Clinical College Clinical Research Ethics Committees (YXLL-2017-02). The study protocol received Ethics Committee approval from all participating centers. Written informed consent was obtained from patients whose competence was established by their accurate orientation for time, place, and person, as well as an understanding of the recruiter's description of the trial or otherwise from their next of kin or their legal representative.

All patients were randomly assigned (1:1) to receive $40 \mathrm{mg} /$ day of rosuvastatin or placebos within 7 days after surgery and 3 days before surgery (Figure 1). Rosuvastatin or placebos were administered via oral. Delirium was assessed twice daily with the Confusion Assessment Method during the first 7 postoperative days. The final follow-up was 30 days after surgery.

\section{Study patients}

Eligible patients were more than 60 years old with joint replacement surgery in ICU. Inclusion criteria were as follows: (1) Aged more than 60 years; (2) Could be randomized and received rosuvastatin or placebo within 10 days perioperative; and (3) under general anesthesia and were admitted to the ICU. Exclusion criteria were as follows: (1) Unsalvageable patients likely on admission; (2) High cholesterol combined with diabetes; (3) Brain injury or neurosurgery; (4) 
Severe sinus bradycardia; (5) Neurologic disease; (6) Abnormal liver enzymes, patients with rhabdomyolysis, and myopathy; (7 Patients with a history of mental illness and epilepsy; (8) Patients with severe lung disease and multiple organ dysfunction; and (9) Researchers found other reasons.

\section{Randomization and masking}

Permuted-block randomization was performed using a computer system with an allocation list generated random numbers (in a 1:1 ratio), using SPSS 14.0 software (SPSS Institute, Hefei, Anhui Medical University). This was performed by a statistician not associated with the project team to protect the blinding and integrity of the study. Results of randomization were sealed in sequentially numbered envelopes and stored at the site of the investigation until the end of the study. During the study period, all included patients were randomly assigned to receive either rosuvastatin (40 mg/day, 10days) or placebos. A study nurse administered the study drugs according to the randomization sequence. Both the study members and patients were blinded to the study drug allocation. If an emergency occurred, like a severe hepatic failure, then two experts could request unmasking of the treatment allocation or adjust or interrupt the study drug if necessary. All situations were documented. Patient demographics, medical histories, and relevant investigation results were collected.

\section{Procedures}

No premedication was administered. All patients underwent a standard preoperative evaluation and were given an American Society of Anesthesiologists (ASA) classification based on their medical comorbidities. All patients received the same general anesthesia protocol. Intravenous (IV) midazolam (1 mg-2 mg) and fentanyl (50 mcg-100 mcg) were given as preoperative sedation and anesthesia was maintained with 3 to $6 \mathrm{mcg} / \mathrm{kg}$ of IV fentanyl. All patients were routinely monitored with electrocardiography (ECG), non-invasive blood pressure, pulse oximetry ( $\mathrm{SpO} 2)$, and Bispectral Index (BIS). Radial arterial pressure and central venous pressures were monitored as necessary.

After surgery, patients were admitted to the ICU and all patients received the same management. Patients in the treatment group received $40 \mathrm{mg}$ /day of rosuvastatin, while the placebo group received an equal Starch piece. All study drugs (rosuvastatin and placebo) were provided from Zhejiang Jingxin Medicine (Co, Ltd, Zhejiang, China). They were dispensed according to the 
randomization results by a pharmacist that did not participate in the rest of the study.

\section{Outcome assessment}

All clinical data were assessed by a masked independent diagnostic and assessment committee. This committee included two researchers that were trained before the study and were not involved in the clinical care of patients. The primary endpoint was the incidence of delirium in the first 7 days after surgery. The first postoperative delirium was evaluated around 24 hours after surgery and evaluated twice daily (from 6 to 8 am and from 6 to 8 pm)[14-16]. Delirium was the assessment by the Confusion Assessment Method (CAM) and the CAM for the Intensive Care Unit (CAM-ICU). Both CAM and CAM-ICU detected 4 features of delirium: (1) Acute onset of mental status changes or a fluctuating course; (2) Inattention; (3) Disorganized thinking; and (4) Altered level of consciousness. To be diagnosed as delirious, a patient displayed features 1 and 2, with either 3 or $4[16]$. For patients that were discharged or died within 7 days after surgery, results of the last delirium assessment were considered the results of the missing data. These patients were excluded when calculating the daily prevalence of delirium in a post-hoc analysis[14]. Second endpoints included all-cause 30-day mortality, length of stay in the ICU, the occurrence of non-delirium postoperative complications, and hospital costs.

\section{Rosuvastatin related adverse events assessment}

The most common adverse events included abnormal liver enzymes, rhabdomyolysis, and myopathy. We need to evaluate all related adverse events and unblind if necessary.

\section{Statistical analysis}

Based on previous data, it was estimated that 739 patients would be required to confirm these effects with an $\alpha$ of $5 \%$ and $80 \%$ power[17]. Further assuming a $10 \%$ loss to follow-up, 845 patients were enrolled. A research nurse entered all baseline and outcome data in the study database. Data were collected on handwritten forms and archived in a password-protected electronic database.

This study described the incidence and relative risk reduction of dichotomous variables for the rosuvastatin -treated group relative to the placebo group, with corresponding 95\% CIs. Demographics and safety data are reported as descriptive statistics (means and standard deviation). Categorical variables were analyzed with the $\chi^{2}$ test, continuity correction $\chi^{2}$ test, or likelihood ratio $\chi 2$ test. Numeric variables were analyzed by use of an unpaired t-test or Mann-Whitney 
U-test. Differences (and 95\% CI for the differences) between the two medians were calculated with the Hodges-Lehmann estimator.

This study did not perform interim analysis. Statistical analyses were done on SPSS 18.0 software (SPSS, Chicago, IL) with two-tailed tests as appropriate. $\mathrm{P}<0.05$ indicates statistical significance. The Clinical Research Ethics Committee from Anhui Medical University, Wuxi clinical college (904th Hospital of Joint Logistic Support Force of PLA), was involved in overseeing the data.

\section{Results}

Between Jan 1, 2017, and Jan 1, 2020, 3512 patients were assessed. A total of 821 were randomly assigned to receive either placebos $(n=411)$ or rosuvastatin $(n=410)$ (Figure 2). There were no lapses in the blinding during the study period. Baseline characteristics of patients showed no statistical significance between the rosuvastatin and placebo groups (Table 1). During the study period, no patients withdrew consent. 35 patients (16 patients in the placebo group and 19 patients in the rosuvastatin group) lost follow-up or clinical data at 30 days. All patients were included in the final intention-to-treat analyses (Figure 2). The final visit of the last randomized patient was done on Oct 31, 2020.

\section{Baseline patient characteristics: Overall population}

3512 patients were enrolled in the present trial, of whom 821 were eligible for this study: 410 (50\%) assigned to the rosuvastatin group and $411(50 \%)$ assigned to the placebo group. The patient's demographics and baseline characteristics had no significant differences between the two groups. The general anesthetic medication, anesthesia time, and operative time were also similar between the two groups. Postoperative medication and management also were similar (Table 1).

\section{The primary endpoint-clinical outcomes}

The incidence of postoperative delirium was $23(5.6 \%)$ of 410 patients in the rosuvastatin group and $42(13.5 \%)$ of 411 patients occurred delirium in the placebo group $(\mathrm{OR}=0.522,95 \% \mathrm{CI}$ $0.308-0.885 ; \mathrm{p}<0.05$, Figure 3$)$.

\section{The secondary endpoint}

Many previous studies had reported that delirium can increase the 30-day all-cause mortality, hospitalization time, and hospitalization expenses. Thus, the present study also evaluated the differences between the two groups in the 30-day all-cause mortality, hospitalization time, and 
hospitalization expenses. According to the 30-day all-cause mortality, 24 (6.1\%) of 391 patients in the rosuvastatin group and $35(8.7 \%)$ of 395 in the placebo group died within 30 days $(\mathrm{OR}=0.67$, $\mathrm{P}=0.147,95 \%$ CI $0.39-1.2$, Figure 4). Hospitalization time in the placebo group was significantly increased, compared to the rosuvastatin group (13.8 \pm 2.5 vs $14.2 \pm 2.8, \mathrm{P}=0.03$, Figure 5). Similarly, hospitalization expenses in the placebo group were significantly higher than the rosuvastatin group (9.3 \pm 2.5 vs $9.8 \pm 2.9, \mathrm{P}=0.007$, Figure 6).

\section{Safety evaluation}

We evaluated the drug-related complications included abnormal liver enzymes and rhabdomyolysis. The occurrence of postoperative abnormal liver enzymes had no statistically significant between the two groups $(9.0 \%$ vs $7.1 \%, \mathrm{P}=0.30, \mathrm{OR}=1.307,95 \%$ CI $0.787-2.169$, Table 2),. The occurrence of rhabdomyolysis in the placebo group and rosuvastatin group showed no differences $(0.73 \%$ vs $0.24 \%, \mathrm{P}=0.37, \mathrm{OR}=3.020,95 \%$ CI $0.31-29.2$, Table 2$)$. Even though, no statistical significance between the two groups on abnormal liver enzymes and rhabdomyolysis, the incidence of postoperative complications in the rosuvastatin group were relatively higher than the placebo group, and need us to attention and avoid serious complications.

\section{Discussion}

The present study found that rosuvastatin can significantly decrease the postoperative delirium after general anesthesia surgery in elderly patients. Meanwhile, rosuvastatin also can reduce the length of hospital stay and hospitalization expenses. For our data, rosuvastatin can not affect 30-day all-cause mortality. While postoperative complications, especially for drug-related complications included abnormal liver enzymes and rhabdomyolysis were similar.

Our study reported that the incidence of postoperative delirium was $13.5 \%$ of placebo patients, and $5.6 \%$ in the rosuvastatin group, The results were similar to a large number of the previous studies[11, 15, 18-20]. Liu[21] reported a retrospective cohort study that included 361 elderly individuals who underwent noncardiac surgery, there were $19.9 \%$ individuals developed postoperative delirium after surgery. Kant[22] also reported a 413 patients study, which indicated that the incidence of postoperative delirium as high as $17 \%$, and preoperative Brain MRI feature may have a predisposition for developing delirium after major surgery. Humeidan[23] reported that the incidence of delirium rate was $23.0 \%$ in the control participants, and the delirium rate in the intervention group was $14.4 \%$. Thus, the incidence of postoperative delirium after general 
anesthesia was extremely high, and it has seriously affected the operative effect and long-term outcome. Besides, postoperative delirium was associated with high morbidity and mortality, worse operative effect, and worse long-term outcome[2, 24]. A previous meta-analysis reported that postoperative delirium can increase the risk of a person experiencing poorer outcomes [25]. Additionally, Postoperative delirium also leads to a longer hospitalization time, and more hospitalization expenses. So, the medication has now become the main treatment.

Many reasons can lead to postoperative delirium, and usually, delirium was a multifactorial disease[2, 26]. Chen[26] reported that major surgery, drugs (sedatives or hypnotics), trauma (especially traumatic brain injury), coma, and sleep deprivation were the most important precipitating factors for delirium by literature review, and summarized the potential mechanisms of delirium, including systemic neuroinflammation, neurotransmitters, cerebral hypoperfusion, micro thrombosis, oxidative stress damage, neuronal apoptosis and neuroprotective, endothelial damage, cerebralvascular spasm and blood-brain barrier injury. How to prevent these mechanisms is the key to the anti-delirium. Statins, as inhibitors of 3-hydroxy-3-methylglutaryl coenzyme A (HMG-CoA) reductase, as its pharmacological mechanism included anti-inflammatory, antioxidative stress, inhibit platelet aggregation, and neuroprotection, it also matched the mechanisms of delirium as the above.

$\mathrm{Yu}[27]$ reported that simvastatin has the potential to be employed as a therapy for depression associated with neuroinflammation, via suppressed the activation of microglia and decreased the expression of proinflammatory cytokines in the hippocampus. Statins can reduce the concentration of glutamate by upregulation of nitric oxide synthase, and Jiang[28] also found that statins can inhibit 5-HT and 5-HTT expression in chronic obstructive pulmonary disease and pulmonary artery hypertension, then it may be ameliorated delirium by regulating neurotransmitters. Many previous studies indicated that statins can alleviate cerebral vasospasm, cerebral perfusion, prevents endothelial dysfunction, and ameliorating neuronal apoptosis and brain injury[7, 9, 10]. Thus, statins can prevent postoperative delirium and within reasonable potential mechanisms.

Redelmeier [29] first reported a large sample study that a retrospective cohort analysis enrolled 284158 consecutive patients aged 65 years and older who were admitted for elective surgery, which showed that statins may be increased the risk of postoperative delirium among the elderly patients. While Katznelson[30] reported another 1059 patients study, it indicated that preoperative 
statins can reduce the odds of postoperative delirium after cardiac surgery with cardiopulmonary bypass. Trezzi[31] reported a retrospective cohort analysis enrolled 12741 cardiac operative patients, the results showed no significant difference between receiving statins group and without statins group after cardiac operation. Oh[15] retrospectively reviewed a large total knee replacement patients under spinal anesthesia, continuous perioperative statin use maybe reduces the risk of delirium after total knee arthroplasty under spinal anesthesia. However, all of these studies were retrospective cohort studies and ultimately did not had a uniform and satisfactory results. For postoperative delirium, there was no high evidence-based medicine and need more prospective randomized controlled studies. In recent studies, most large prospective randomized controlled studies explore early administration of statins in the prevention and treatment of delirium in critically ill patients or undergoing mechanical ventilation[13, 17, 19, 32]. Thus, no related large sample prospective randomized controlled trials to explore the role of perioperative statins for postoperative delirium after elderly general anesthesia patients.

The present study firstly demonstrated that perioperative rosuvastatin treatment can reduce the incidence of delirium after elective general anesthesia operation, also can decrease the length of stay in the ICU and hospital costs. Additionally, rosuvastatin did not increased the drug-related complications. It was masked and enrolled 821 patients, most patients finished the follow-up. Another strength in this trial was a double-blind, randomized, and placebo-controlled design (The study was registered with http://www.chictr.org.cn, number: ChiCTR-IPR-17011984). There were several limitations of this study and need to improve: This study should analyze more clinical factors, such as sleep quality, pain, etc. The follow-up time in this study was too short and need long-term follow-up, the effects on long-term activities of daily living [33] and cognitive functions also were unclear. This study used a single dose of statins $(40 \mathrm{mg} /$ day), the conventional low dose may not work well.

\section{Conclusions}

In this present study, we found that administration of perioperative rosuvastatin can reduce the incidence of delirium after elective general anesthesia operation in elderly patients, also can decrease the length of stay in the ICU and hospital costs. No benefit for clinical outcome, 30-day all-cause mortality after perioperative rosuvastatin treatment. The effects of longer-term or larger 
doses $(80 \mathrm{mg} /$ day $)$ of rosuvastatin remain unknown. The effects on long-term activities of daily living and cognitive functions also were unclear. Further investigation of elderly patients undergoing elective general anesthesia operation and different doses are needed to fully understand the potential usefulness of rosuvastatin for postoperative delirium.

\section{Supplementary files}

The consort 2010 checklist

\section{Abbreviations}

ASA: American Society of Anesthesiology; BIS: Bispectral Index; CAM: Confusion Assessment Method; ECG: electrocardiography; HMG-CoA: 3-hydroxy-3-methylglutaryl coenzyme A; ICU: Intensive Care Unit; IV: Intravenous; SpO2: pulse oximetry

\section{Declarations}

\section{Acknowledgements}

Not applicable.

\section{Statement for guidelines}

The study was performed in accordance with the ethical standards of the Declaration of Helsinki (1964) and its subsequent amendments.

\section{Authors' contributions}

All authors have read and approved the manuscript. XX and LX helped design the study, conduct the study, collect the data, analyze the data, and prepare the manuscript. LJ, TH, and LW helped analyze the data, and prepare the manuscript. XX and LX helped conduct the study, collect the data, analyze the data, and prepare the manuscript. TH and LW helped conduct the study and collect the data. XX and LX helped conduct the study and collect the data.

\section{Funding}

Not applicable. 


\section{Availability of data and materials}

The datasets used and analysed during the current study are available from the corresponding author on reasonable request.

\section{Ethics approval and consent to participate}

The study protocol was approved by the Anhui Medical University Affiliated Wuxi Clinical College Clinical Research Ethics Committees (ChiCTR-IPR-17011984, YXLL-2017-02). The study protocol received Ethics Committee approval from all participating centers. Written informed consent was obtained from patients whose competence was established by their accurate orientation for time, place, and person, as well as an understanding of the recruiter's description of the trial or otherwise from their next of kin or their legal representative.

\section{Consent for publication}

Not applicable.

\section{Competing interests}

The authors declare that they have no competing interests.

\section{Author details}

1 Department of Anesthesiology, Wuxi Clinical College of Anhui Medical University, $904^{\text {th }}$ Hospital of Joint Logistic Support Force of PLA, Wuxi Clinical College of Anhui Medical University, Wuxi, 214000.

Reference:

1. Gleason LJ, Schmitt EM, Kosar CM, Tabloski P, Saczynski JS, Robinson T, Cooper Z, Rogers SO, Jr., Jones RN, Marcantonio ER et al: Effect of Delirium and Other Major Complications on Outcomes After Elective Surgery in Older Adults. JAMA Surg 2015, 150(12):1134-1140.

2. Inouye SK, Westendorp RG, Saczynski JS: Delirium in elderly people. Lancet 2014, 383(9920):911-922.

3. Redelmeier DA, Manzoor F, Thiruchelvam D: Association Between Statin Use and Risk 
of Dementia After a Concussion. JAMA Neuro/ 2019, 76(8):887-896.

4. Girard TD, Exline MC, Carson SS, Hough CL, Rock P, Gong MN, Douglas IS, Malhotra A, Owens RL, Feinstein DJ et al: Haloperidol and Ziprasidone for Treatment of Delirium in Critical Illness. N Eng/ J Med 2018, 379(26):2506-2516.

5. van den Boogaard M, Slooter AJC, Brüggemann RJM, Schoonhoven L, Beishuizen A, Vermeijden JW, Pretorius D, de Koning J, Simons KS, Dennesen PJW et al: Effect of Haloperidol on Survival Among Critically III Adults With a High Risk of Delirium: The REDUCE Randomized Clinical Trial. Jama 2018, 319(7):680-690.

6. Avidan MS, Maybrier HR, Abdallah AB, Jacobsohn E, Vlisides PE, Pryor KO, Veselis RA, Grocott HP, Emmert DA, Rogers EM et al: Intraoperative ketamine for prevention of postoperative delirium or pain after major surgery in older adults: an international, multicentre, double-blind, randomised clinical trial. Lancet 2017, 390(10091):267-275.

7. Chen JH, Yang LK, Chen L, Wang YH, Wu Y, Jiang BJ, Zhu J, Li PP: Atorvastatin ameliorates early brain injury after subarachnoid hemorrhage via inhibition of AQP4 expression in rabbits. Int J Mol Med 2016, 37(4):1059-1066.

8. Chen J, Li M, Zhu X, Chen L, Yang S, Zhang C, Wu T, Feng X, Wang Y, Chen Q: Atorvastatin reduces cerebral vasospasm and infarction after aneurysmal subarachnoid hemorrhage in elderly Chinese adults. Aging (Albany NY) 2020, 12(3):2939-2951.

9. Chen JH, Wu T, Xia WY, Shi ZH, Zhang CL, Chen L, Chen QX, Wang YH: An early neuroprotective effect of atorvastatin against subarachnoid hemorrhage. Neural Regen Res 2020, 15(10):1947-1954

10. Chen JH, Wu T, Yang LK, Chen L, Zhu J, Li PP, Hu X, Wang YH: Protective effects of atorvastatin on cerebral vessel autoregulation in an experimental rabbit model of subarachnoid hemorrhage. Mol Med Rep 2018, 17(1):1651-1659.

11. Lee DS, Lee MY, Park CM, Kim DI, Kim YW, Park YJ: Preoperative statins are associated with a reduced risk of postoperative delirium following vascular surgery. PLOS One 2018, 13(3):e0192841.

12. Martínez-Comendador J, Alvarez JR, Sierra J, Teijeira E, Adrio B: Preoperative statin therapy in cardiac surgery is more effective in patients who display preoperative activation of the inflammatory system. Tex Heart Inst J 2013, 40(1):42-49.

13. Needham DM, Colantuoni E, Dinglas VD, Hough CL, Wozniak AW, Jackson JC, Morris PE, Mendez-Tellez PA, Ely EW, Hopkins RO: Rosuvastatin versus placebo for delirium in intensive care and subsequent cognitive impairment in patients with sepsis-associated acute respiratory distress syndrome: an ancillary study to a randomised controlled trial. Lancet Respir Med 2016, 4(3):203-212.

14. Su X, Meng ZT, Wu XH, Cui F, Li HL, Wang DX, Zhu X, Zhu SN, Maze M, Ma D: Dexmedetomidine for prevention of delirium in elderly patients after non-cardiac surgery: a randomised, double-blind, placebo-controlled trial. Lancet 2016, 388(10054):1893-1902.

15. Oh TK, Park HY, Shin HJ, Jeon YT, Do SH, Hwang JW: The Role of Perioperative Statin Use in the Prevention of Delirium After Total Knee Replacement Under Spinal Anesthesia. J Arthroplasty 2018, 33(12):3666-3671.e3661. 
16. Xuan Y, Fan R, Chen JH, Wang YH, Wu JY, Yang JJ, Luo YC: Effects of dexmedetomidine for postoperative delirium after joint replacement in elderly patients: a randomized, double-blind, and placebo-controlled trial. International Journal of Clinical and Experimental Medicine 2018, 11(12):13147-13157.

17. Page VJ, Davis D, Zhao XB, Norton S, Casarin A, Brown T, Ely EW, McAuley DF: Statin use and risk of delirium in the critically ill. Am J Respir Crit Care Med 2014, 189(6):666-673.

18. Burry L, Hutton B, Williamson DR, Mehta S, Adhikari NK, Cheng W, Ely EW, Egerod I, Fergusson DA, Rose L: Pharmacological interventions for the treatment of delirium in critically ill adults. Cochrane Database Syst Rev 2019, 9(9):Cd011749.

19. Page VJ, Casarin A, Ely EW, Zhao XB, McDowell C, Murphy L, McAuley DF: Evaluation of early administration of simvastatin in the prevention and treatment of delirium in critically ill patients undergoing mechanical ventilation (MoDUS): a randomised, double-blind, placebo-controlled trial. Lancet Respir Med 2017, 5(9):727-737.

20. Mather JF, Corradi JP, Waszynski C, Noyes A, Duan Y, Grady J, Dicks R: Statin and Its Association With Delirium in the Medical ICU. Crit Care Med 2017, 45(9):1515-1522.

21. Liu H, Dai M, Guan H, Gao X, Zhou Y, Sun X, Zhou J, Hu X, Li X, Song Y et al: Preoperative Prognostic Nutritional Index Value is Related to Postoperative Delirium in Elderly Patients After Noncardiac Surgery: A Retrospective Cohort Study. Risk Manag Healthc Policy 2021, 14:1-8.

22. Kant IMJ, de Bresser J, van Montfort SJT, Mutsaerts H, Witkamp TD, Buijsrogge M, Spies C, Hendrikse J, Slooter AJC: Preoperative brain MRI features and occurrence of postoperative delirium. J Psychosom Res 2021, 140:110301.

23. Humeidan ML, Reyes JC, Mavarez-Martinez A, Roeth C, Nguyen CM, Sheridan E, Zuleta-Alarcon A, Otey A, Abdel-Rasoul M, Bergese SD: Effect of Cognitive Prehabilitation on the Incidence of Postoperative Delirium Among Older Adults Undergoing Major Noncardiac Surgery: The Neurobics Randomized Clinical Trial. JAMA Surg 2020

24. Oh ES, Fong TG, Hshieh TT, Inouye SK: Delirium in Older Persons: Advances in Diagnosis and Treatment. Jama 2017, 318(12):1161-1174.

25. Scott JE, Mathias JL, Kneebone AC: Incidence of delirium following total joint replacement in older adults: a meta-analysis. Gen Hosp Psychiatry 2015, 37(3):223-229.

26. Chen J, Wang Y, Hu X, Li M, Xiong K, Zhang Z, Chen Q: The role of statins in the management of delirium: Recent advances. CNS Neurol Disord Drug Targets 2020.

27. Yu XB, Zhang HN, Dai Y, Zhou ZY, Xu RA, Hu LF, Zhang CH, Xu HQ, An YQ, Tang CR et al: Simvastatin prevents and ameliorates depressive behaviors via neuroinflammatory regulation in mice. J Affect Disord 2019, 245:939-949.

28. Jiang X, Yuan L, Li P, Wang J, Wang P, Zhang L, Sun B, Sun W: Effect of Simvastatin on 5-HT and 5-HTT in a Rat Model of Pulmonary Artery Hypertension. Cell Physiol Biochem 2015, 37(5):1712-1724.

29. Redelmeier DA, Thiruchelvam D, Daneman N: Delirium after elective surgery among elderly patients taking statins. Cmaj 2008, 179(7):645-652.

30. Katznelson R, Djaiani GN, Borger MA, Friedman Z, Abbey SE, Fedorko L, Karski J, Mitsakakis N, Carroll J, Beattie WS: Preoperative use of statins is associated with 
reduced early delirium rates after cardiac surgery. Anesthesiology 2009, 110(1):67-73.

31. Trezzi M, Blackstone EH, Sun Z, Li L, Sabik JF, 3rd, Lytle BW, Gordon SM, Koch CG: Statin therapy is associated with fewer infections after cardiac operations. Ann Thorac Surg 2013, 95(3):892-900.

32. Morandi A, Hughes CG, Thompson JL, Pandharipande PP, Shintani AK, Vasilevskis EE, Han JH, Jackson JC, Laskowitz DT, Bernard GR et al: Statins and delirium during critical illness: a multicenter, prospective cohort study. Crit Care Med 2014, 42(8):1899-1909.

33. Kothari V, Stevens RJ, Adler Al, Stratton IM, Manley SE, Neil HA, Holman RR, Grp UKPDS: UKPDS 60 - Risk of stroke in type 2 diabetes estimated by the UK Prospective Diabetes Study risk engine. Stroke 2002, 33(7):1776-1781.

Figure legend

Figure 1. Study design.

Figure 2. Trial profile.

Figure 3. The incidence of postoperative delirium. The odds ratios percentages are rounded. Event rate (\%) for postoperative delirium. $\mathrm{OR}=0.522,95 \%$ CI $0.308-0.885 ; \mathrm{p}<0.05$.

Figure 4. 30-day all-cause mortality. The odds ratios percentages are rounded. Event rate (\%) for 30-day all-cause mortality. $\mathrm{OR}=0.67, \mathrm{P}=0.147,95 \% \mathrm{CI} 0.39-1.2$.

Figure 5. Hospitalization time. Hospitalization time in the rosuvastatin group was significantly decreased more than in the place group $(\mathrm{P}=0.03)$.

Figure 6. Hospitalization cost. Hospitalization cost in the rosuvastatin group was significantly decreased more than in the place group $(\mathrm{P}=0.007)$.

Table Legend

Table 1. Demographic and baseline characteristics of the study population in the two groups.

Table 2. Comparison of postoperative complications between the two groups. 
Figures

30-day all-cause mortality hospitalization time

Randomisation(1:1, rosuvastatin:placebos) hospitalization expenses

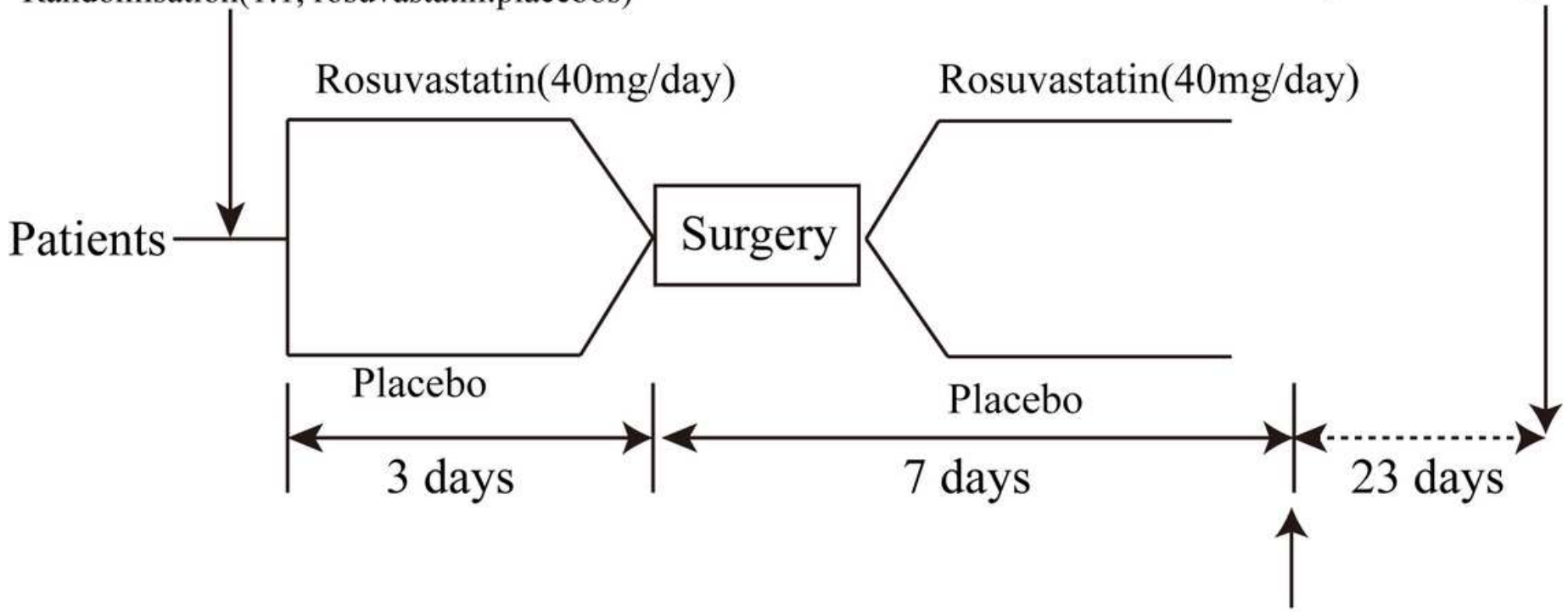

Delirium

Complications

\section{Figure 1}

Study design. 


\section{2 patients assessed}
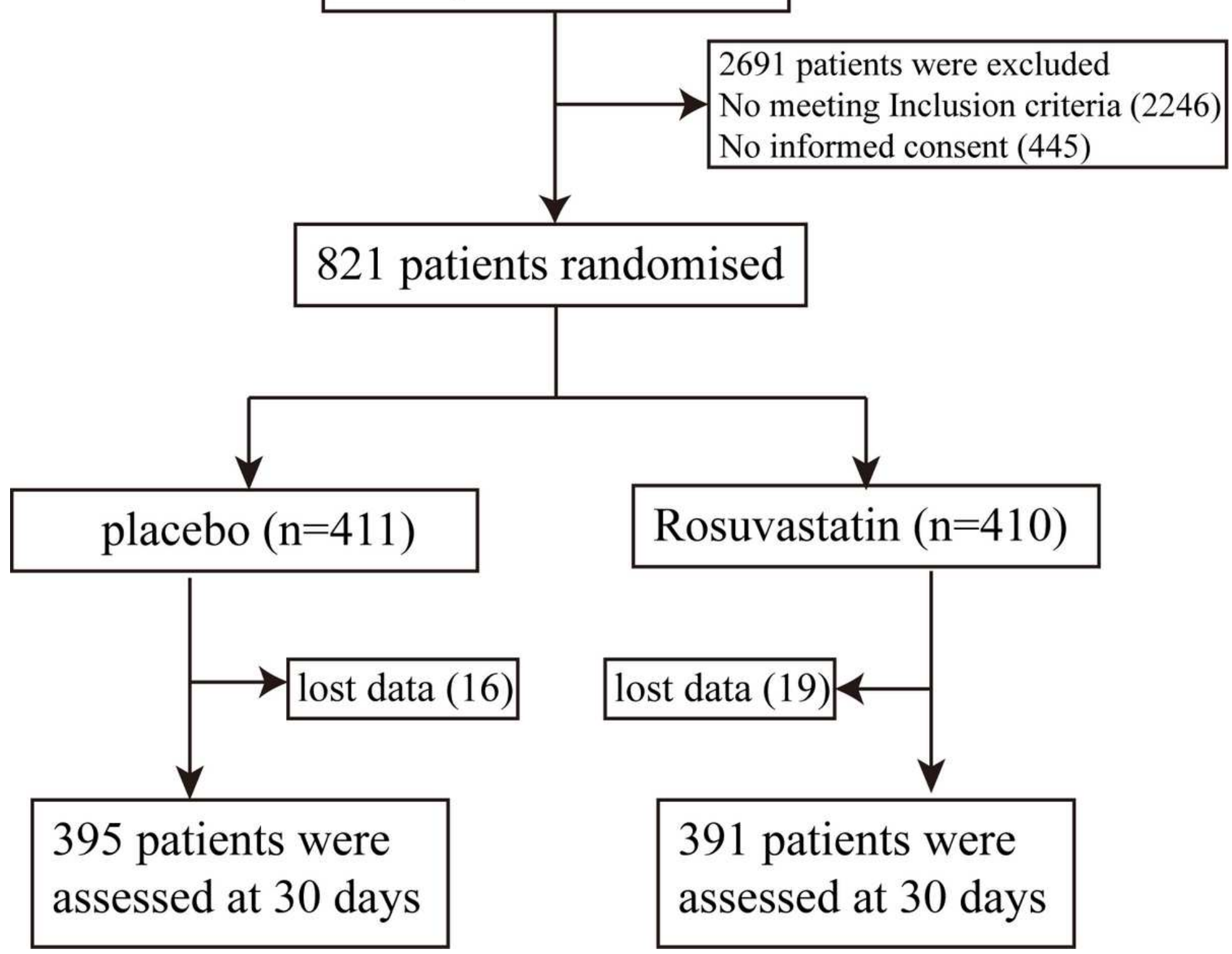

Figure 2

Trial profile. 


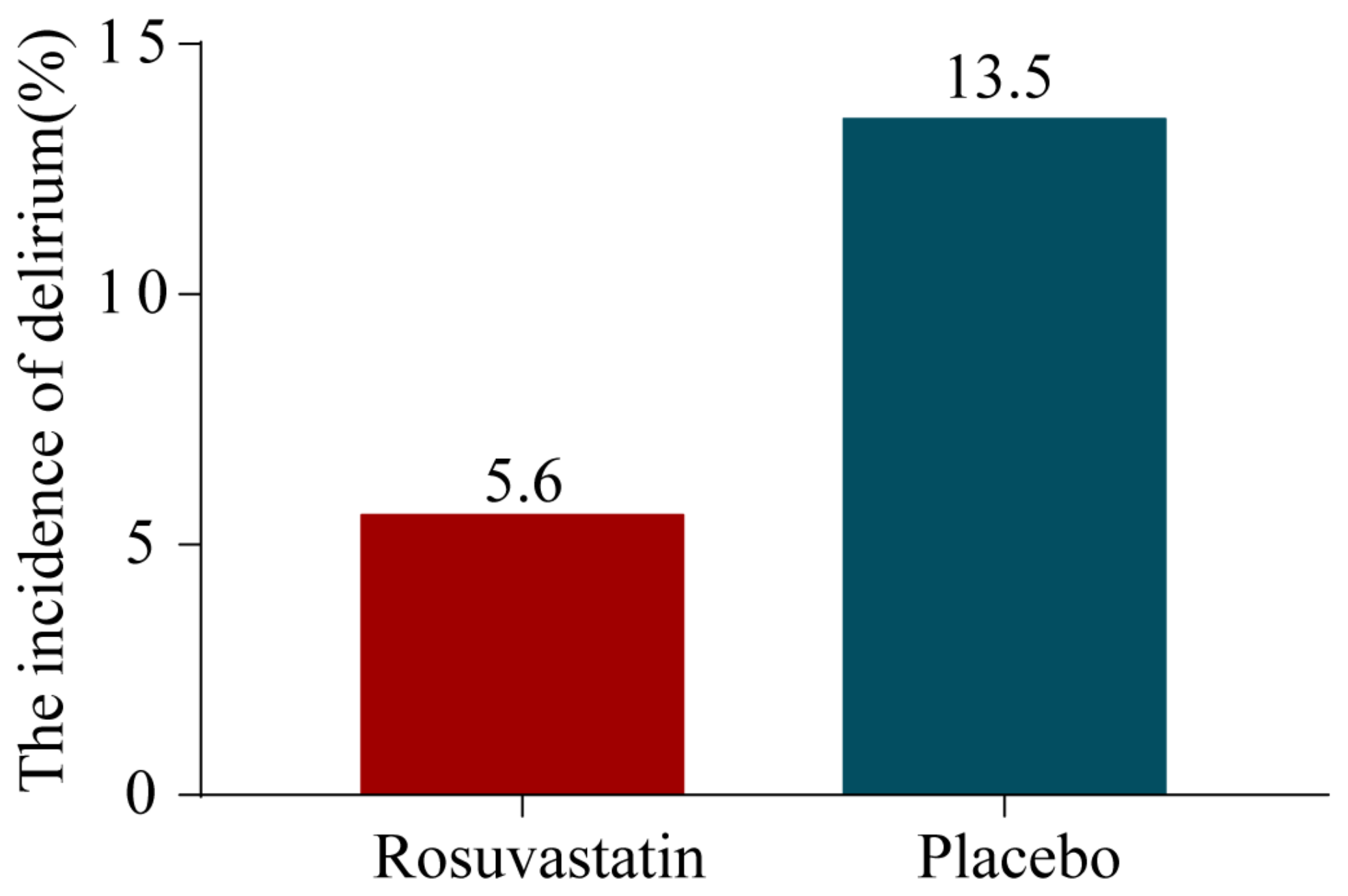

\section{$\mathrm{OR}=0.522 \quad 95 \%$ CI $0.308-0.885 \quad \mathrm{p}<0.05$}

Figure 3

The incidence of postoperative delirium. The odds ratios percentages are rounded. Event rate (\%) for postoperative delirium. $\mathrm{OR}=0.522,95 \% \mathrm{Cl} 0.308-0.885 ; \mathrm{p}<0.05$. 


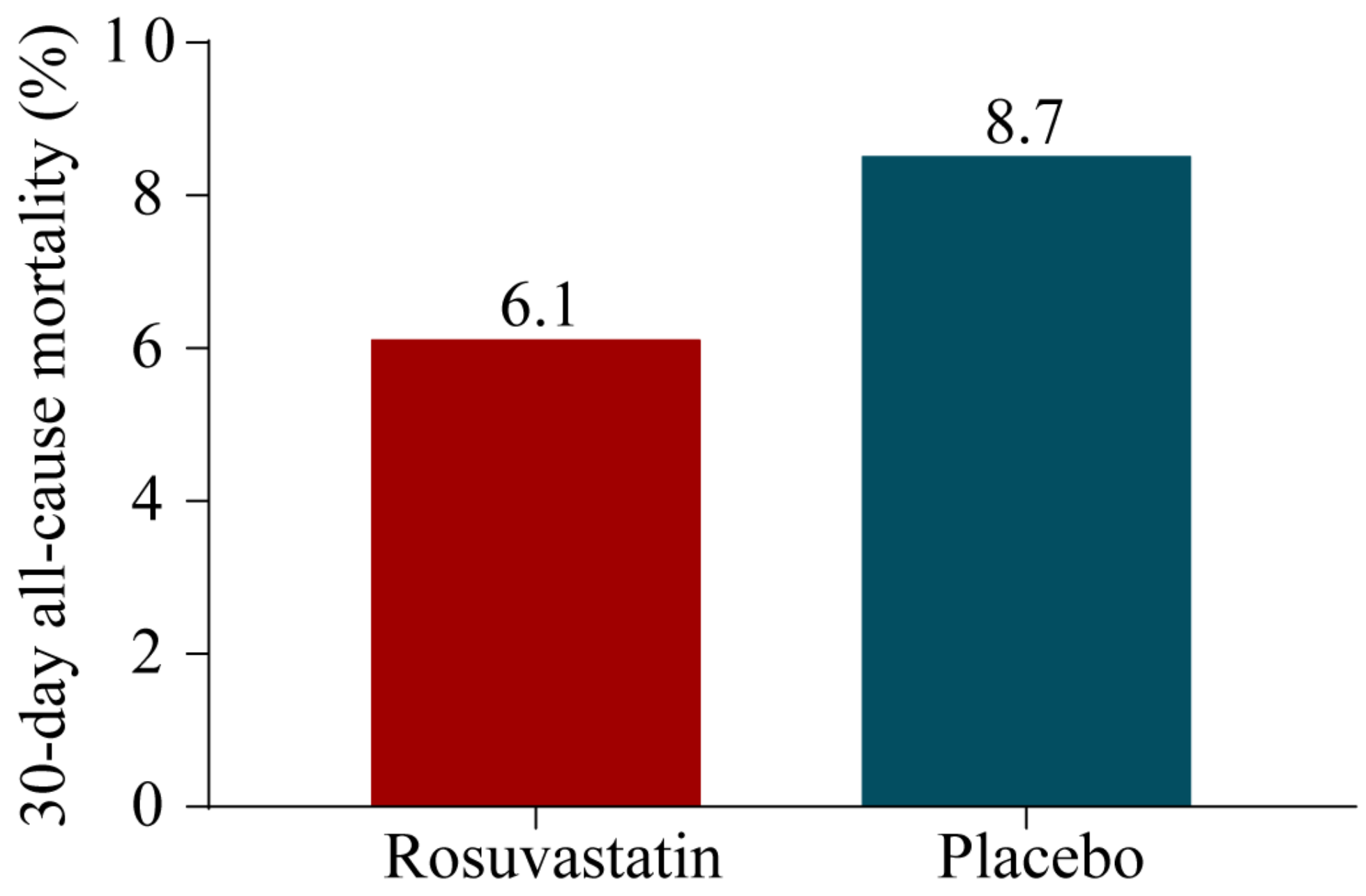

\section{$\mathrm{OR}=0.670 \quad 95 \%$ CI $0.39-1.20 \quad \mathrm{p}=0.147$}

Figure 4

30-day all-cause mortality. The odds ratios percentages are rounded. Event rate (\%) for 30-day all-cause mortality. OR=0.67, $\mathrm{P}=0.147,95 \% \mathrm{Cl} 0.39-1.2$. 


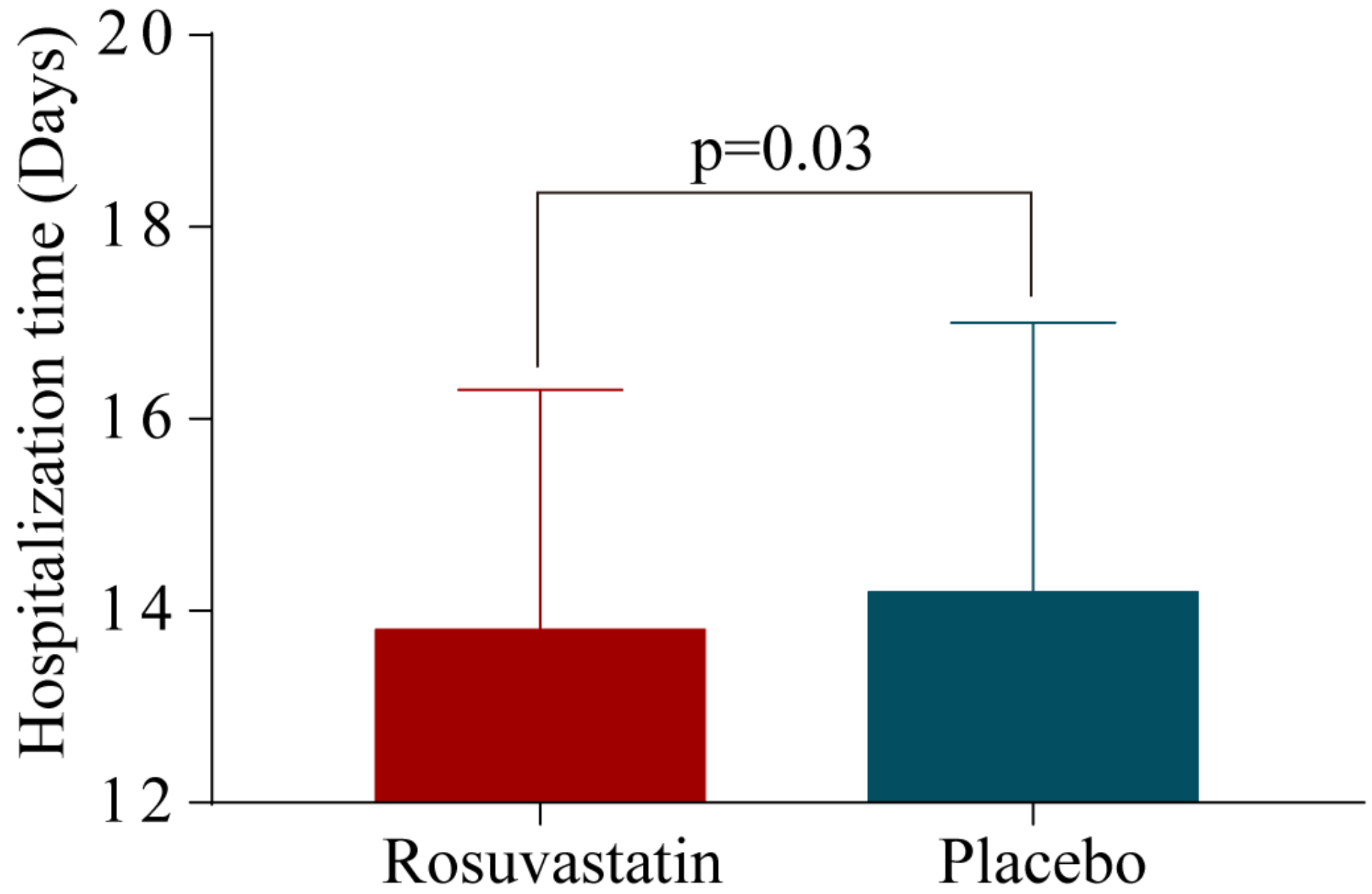

Figure 5

Hospitalization time. Hospitalization time in the rosuvastatin group was significantly decreased more than in the place group $(P=0.03)$. 


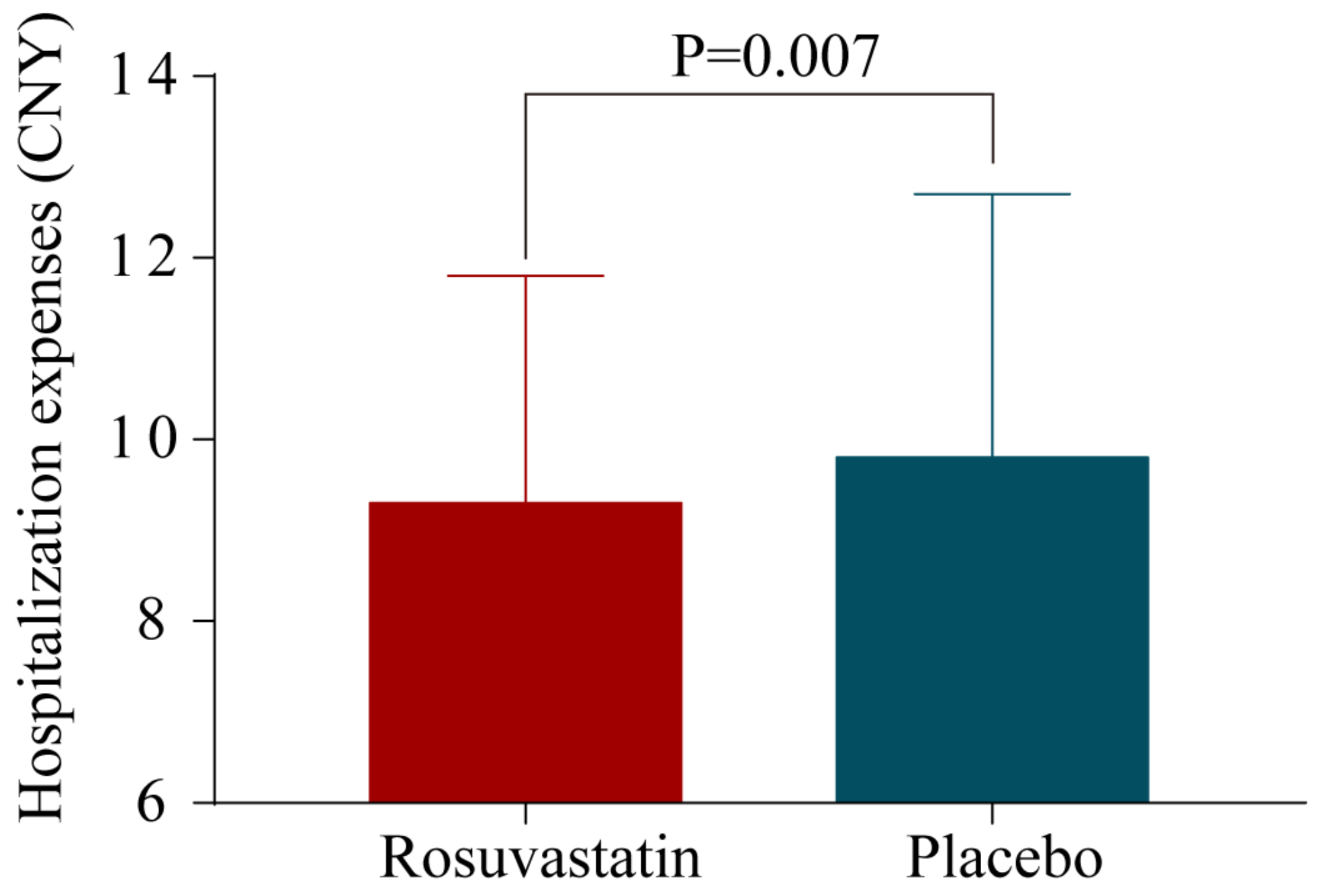

Figure 6

Hospitalization cost. Hospitalization cost in the rosuvastatin group was significantly decreased more than in the place group $(\mathrm{P}=0.007)$. 